\title{
Alignment of the grating wheel mechanism for a ground-based, cryogenic, near-infrared astronomy instrument
}

\author{
Sharon M. Gutkowski ${ }^{a}$, Raymond G. Ohl ${ }^{{ }^{a}}$, Jason E. Hylan ${ }^{b}$, John G. Hagopian ${ }^{\mathrm{a}}$, Stephen E. Kraft ${ }^{\mathrm{a}}$, \\ J. Eric Mentzell ${ }^{a}$, Joseph A. Connelly ${ }^{a}$, Joseph P. Schepis ${ }^{a}$, Leroy M. Sparr ${ }^{a}$, Matthew A. \\ Greenhouse $^{a}$, John W. MacKenty ${ }^{c}$ \\ ${ }^{a}$ NASA/Goddard Space Flight Center, Greenbelt, Md. 20771 \\ bswales Aerospace, Inc., Beltsville, Md. 20705 \\ ${ }^{\mathrm{c}}$ Space Telescope Science Institute, Baltimore, Md. 21218
}

\begin{abstract}
We describe the population, optomechanical alignment, and alignment verification of near-infrared gratings on the grating wheel mechanism (GWM) for the Infrared Multi-Object Spectrometer (IRMOS). IRMOS is a cryogenic (80 K), principle investigator-class instrument for the $2.1 \mathrm{~m}$ and Mayall $3.8 \mathrm{~m}$ telescopes at Kitt Peak National Observatory, and a MEMS spectrometer concept demonstrator for the James Webb Space Telescope.
\end{abstract}

The GWM consists of 13 planar diffraction gratings and one flat imaging mirror $(58 \times 57 \mathrm{~mm})$, each mounted at a unique compound angle on a $32 \mathrm{~cm}$ diameter gear. The mechanism is predominantly made of Al 6061 . The grating substrates are stress relieved for enhanced cryogenic performance. The optical surfaces are replicated from off-the-shelf masters. The imaging mirror is diamond turned. The GWM spans a projected diameter of $\sim 48 \mathrm{~cm}$ when fully assembled, utilizes several flexure designs to accommodate potential thermal gradients, and is controlled using custom software with an off-the-shelf controller.

Under ambient conditions, each grating is aligned in six degrees of freedom relative to a coordinate system that is referenced to an optical alignment cube mounted at the center of the gear. The local tip/tilt $\left(R_{x} / R_{y}\right)$ orientation of a given grating is measured using the zero-order return from an autocollimating theodolite. The other degrees of freedom are measured using a two-axis cathetometer and rotary table. Each grating's mount includes a one-piece shim located between the optic and the gear. The shim is machined to fine align each grating. We verify ambient alignment by comparing grating diffractive properties to model predictions.

Keywords: IRMOS, optomechanical, alignment, integration, mechanism, grating, wheel

\section{INTRODUCTION}

The Infrared Multi-Object Spectrometer (IRMOS) is a ground-based, cryogenic $(80 \mathrm{~K})$ instrument for use on the $2.1 \mathrm{~m}$ and Mayall $3.8 \mathrm{~m}$ telescopes at Kitt Peak National Observatory (KPNO) ${ }^{1,2}$ This principle investigator-class instrument is scheduled to begin its 5-year mission in fall 2003. In addition to its scientific role, IRMOS is a microelectromechanical systems (MEMS) spectrometer concept demonstrator for the James Webb Space Telescope (JWST) project at NASA/Goddard Space Flight Center (GSFC). IRMOS is a collaboration of the Space Telescope Science Institute (STScI), GSFC, and KPNO, lead by Dr. John W. MacKenty of STScI.

IRMOS is capable of observing the spectra of $\sim 100$ objects per observation in its $2.8 \times 2.0$ arcmin field of view (3.8 m telescope) with low- to mid-resolving power $(R=\lambda \Delta \lambda=300-3000)$. The instrument's wavelength range encompasses the $\mathrm{Z}, \mathrm{J}, \mathrm{H}$ and $\mathrm{K}$ spectral bands $(0.8-2.5 \mu \mathrm{m})$. IRMOS employs two cryogenic, science mode-select mechanisms -- a filter wheel and a grating wheel mechanism (GWM). We describe the GWM design, pre-population optical measurements, and optomechanical alignment of the optics on the GWM under ambient conditions. We also cover ambient alignment verification.

\footnotetext{
- Correspondence: Email: Raymond.G.Ohl@nasa.gov; tel.: 301-286-8368; fax: 301-286-0204
} 


\section{GRATING WHEEL MECHANISM DESIGN}

The purpose of the GWM is to allow the IRMOS user to select one of 13 planar diffraction gratings to generate spectra or a flat mirror for images of astronomical targets. The GWM rotates the desired optic to the "active" position on the wheel relative to the IRMOS optical bench. During an observation, only the desired grating/mirror is a part of the optical path --- the other gratings are covered by a baffle. The gratings and mirror are mounted on a large gear $(\sim 32 \mathrm{~cm}$ diameter) at a unique compound angle set by a custom carrier (Figure 1). The gear is attached to an axle via flexures. The gear is driven by a stepper motor via an anti-backlash pinion. There are 2000 steps in one complete wheel rotation $\left(0.18^{\circ}\right.$ per step). We use custom control software and an off-the-shelf controller to drive the GWM." Schepis et al. (2003) discuss GWM mechanical requirements, design, and component-level cryogenic performance. ${ }^{3}$

CAD views of the GWM are shown in Figure 1. The GWM spans a projected diameter of $\sim 48 \mathrm{~cm}$ when fully populated with gratings and carriers, and rotates about the center of the large wheel (cube $\mathrm{Cl}$ ). There are a total of 14 grating carriers: one for each grating (13) and one for the imaging mirror. Each carrier is unique --- the gratings have different orientations in the instrument optical path and the carriers place the gratings at approximately the design orientation. The darker shaded carrier shown in Figure 1 is in the GWM's "active" position. When a grating is rotated into this position, IRMOS produces spectra of the objects in the field of view. When the imaging mirror is in the active position (as shown in Figure 1), the mechanism is at the "home" position. A voltage-monitoring sensor finds the home position by locating a ferrous target on the rear of the wheel and peaking up on the voltage difference.

We define two Cartesian coordinate systems on the GWM for alignment purposes (Figure 1). Each grating (or the mirror) has a local coordinate system (LCS), centered on the optical aperture, with the z-axis pointing into the substrate and the $\mathrm{x}$-axis parallel to the dispersion direction (i.e., the $\mathrm{y}$-axis is parallel to the lines on the grating). The mechanism coordinate system (MCS) has origin at the center of the large gear and has z-axis pointing away from the face of the wheel and parallel to the axis of rotation. The MCS y-axis points toward the stepper motor when the wheel is oriented such that the imaging mirror is in the active position (i.e., the GWM is at "home"). The MCS does not rotate with the wheel/gear.

\subsection{Mechanical requirements}

The rotational repeatability tolerance of the GWM in MCS Rz (i.e., the ability of the GWM to repeat a wheel position) is \pm 15 arcsec. Repeatability, "backlash," and "wobble" testing is discussed in Section 3.1. Schepis et al. (2003) discuss mechanical tolerances in more detail. ${ }^{3}$

\subsection{Optical alignment requirements}

The gratings and imaging mirror must be aligned in the MCS to better than \pm 1 arcmin in tip/tilt $\left(R_{x} / R_{y}\right)$ and \pm 6 arcmin in clocking $\left(R_{z}\right)$. Translation tolerances in $x$ and $y$ are $\pm 1 \mathrm{~mm}$ and $\pm 2 \mathrm{~mm}$ in $\mathrm{z}$ ( $\sim$ focus). These allocations were generated by an optical tolerance analysis for the instrument under the assumption of negligible alignment error of the GWM assembly to the instrument bench coordinate system and negligible unanticipated alignment change from ambient to the operating temperature $(80 \mathrm{~K})$.

\footnotetext{
- Galil Motion Control, 3750 Atherton Rd., Rocklin, California 95765; tel.: 916-626-0101
} 


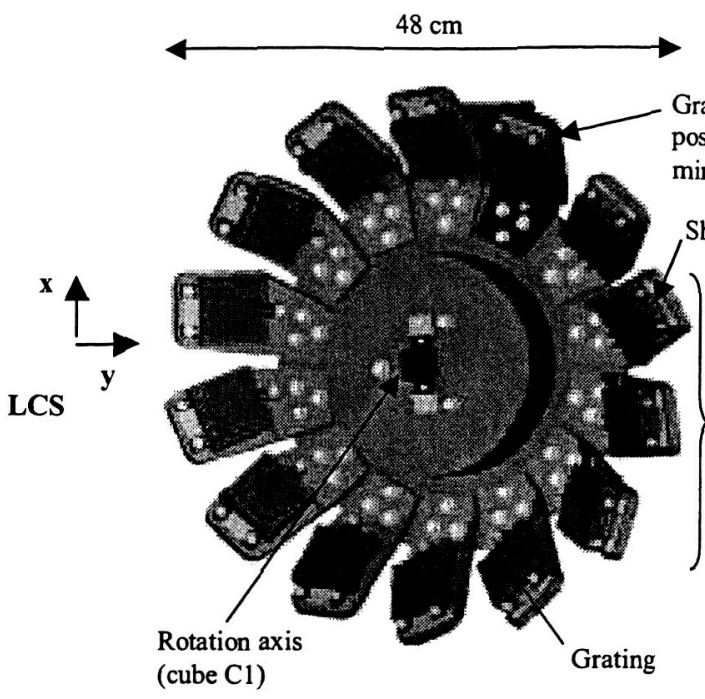

(a)

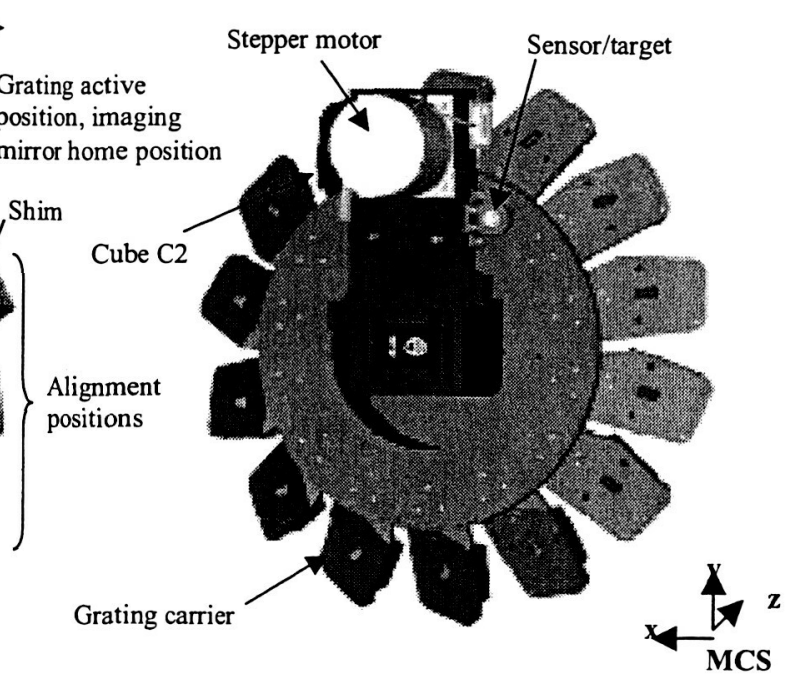

(b)

Figure 1. CAD software model views of (a) front of GWM, and (b) rear of GWM. Approximate orientations of a grating's local coordinate system (LCS) and the mechanism coordinate system (MCS) are shown.

\subsection{Optical prescriptions}

Grating and mirror optical prescriptions and wheel positions are listed in Table 1. All substrates are stress-relieved Al $6061^{4}$ and have an aspect ratio of $\sim 6: 1$. The mount is a semi-kinematic, three-point flexure design. ${ }^{5,6}$ The planar gratings were replicated from an off-the-shelf master and gold-coated for greater IR reflectivity." Zero-order figure tests show that the gratings meet their figure error requirement of $<0.1 \lambda$ RMS $(\lambda=632.8 \mathrm{~nm})$. The imaging mirror was diamond turned flat for a figure error of $<0.1 \lambda$ RMS $(\lambda=632.8 \mathrm{~nm}){ }^{\dagger}$ post-polished at GSFC for a microroughness of $<3 \mathrm{~nm}$ RMS, ${ }^{7}$ and gold-coated. ${ }^{\ddagger}$

Each substrate incorporates alignment fiducials to facilitate optomechanical alignment without reference to the front, diffractive optical surface. ${ }^{5}$ The rear surface of the substrate is diamond turned flat (best effort surface error tolerances). Thin $(\sim 0.125 \mathrm{~mm}), \sim 6 \mathrm{~mm}$ diameter, crosshair-like targets are inscribed on this rear datum surface: One fiducial is located at the center of the clear aperture. Two are located on a line through the center of the aperture and parallel to the dispersion direction. Another crosshair fiducial is located on the center of each of three side faces of the substrate. After grating fabrication, we measure the as-built orientation of these fiducials in six degrees of freedom with respect to the active optical surface: Using autocollimating theodolites, the pointing direction of the normal to the rear surface is measured with respect to the direction of the zero order $\left(\operatorname{LCS} R_{x}\right.$ and $\left.R_{y}\right)$. We measure the clocking of the line of three cross fiducials with respect to the dispersion direction by aiming a low power, $\lambda=632.8 \mathrm{~nm}$ laser normal to the front optical surface and observing the location of the diffracted orders on a ground-glass sheet using a cathetometer mounted behind the grating such that it can also observe the line of cross fiducials (LCS $R_{z}$ ). Also using the cathetometer, we measure the as-built location of the center cross fiducial with respect to the clear aperture (LCS $x$ and $y$ ) and the side fiducials with respect to the front optical surface (LCS z).

- Richardson Gratings, 705 St. Paul Street, Rochester, New York 14605; tel.: 585-262-1331

${ }^{\dagger}$ Labor fuer Mikrozerspanung, Universitaet Bremen, Badgasteiner Str. 2, 28359 Bremen, Germany; tel.: 421-218-2318

‡ Janos Technology, Inc., 1068 Grafton Road, Townshend, Vermont 05353-7702, USA; tel.: 802-365-7714 
Table 1. Grating list

\begin{tabular}{|c|c|c|c|c|c|}
\hline $\begin{array}{c}\text { Mechanism } \\
\text { Position } \\
\text { Number }\end{array}$ & $\begin{array}{c}\text { Mechanism } \\
\text { Position Name }\end{array}$ & Grating Name & Lines/mm & $\begin{array}{c}\text { Blaze } \\
\text { Angle } \\
\text { (degrees) }\end{array}$ & $\begin{array}{c}\text { Ist Order } \\
\text { Littrow } \\
(\boldsymbol{\mu m})\end{array}$ \\
\hline 1 & 1 & low res J & 36.152 & 1.4 & 1.3 \\
\hline 2 & 2 & low res H & 45 & 2.22 & 1.75 \\
\hline 3 & 3 & low res K & 35 & 2.3 & 2.3 \\
\hline 4 & 4 & mid res J & 235.8 & 9.25 & 1.31 \\
\hline 5 & 5 & mid res H & 150 & 5.4 & 1.25 \\
\hline 6 & 6 & mid res K & 100 & 7.2 & 2.5 \\
\hline 7 & 7 & high res J (blue) & 600 & 22 & 1.2 \\
\hline 8 & 8 & high res J (red) & 600 & 22 & 1.2 \\
\hline 9 & 9 & high res H (blue) & 400 & 18.6 & 1.6 \\
\hline 10 & 10 & high res H (red) & 400 & 18.6 & 1.6 \\
\hline 11 & 11 & high res K (blue) & 300 & 17.5 & 2 \\
\hline 12 & 12 & high res K (red) & 300 & 22 & 2.5 \\
\hline 13 & PARK (transport) & Z & 300 & 8.6 & 1 \\
\hline 14 & HOME & Imaging Mirror (IM) & n/a & n/a & n/a \\
\hline
\end{tabular}

\subsection{Population plan}

For each position, the GWM wheel must be populated with a carrier, shim, and grating or mirror. Population and alignment proceeds as follows: First, the carriers are rough-aligned to an ideal MCS (i.e., we assume that the alignment cube $\mathrm{Cl}$ has front face normal to the axis of rotation; Section 3.2). Second, the shims and gratings are attached, but not aligned. The as-built MCS is defined based on the measured pointing direction of the axis of wheel rotation (Section 4.2). This is performed with the carriers, shims, and gratings attached, but not aligned, so that the axis of rotation has its final pointing direction (they add significant mass to the wheel). Third, the gratings are fine-aligned to the MCS in six degrees of freedom (Section 4.3). Finally, alignment is verified using the diffractive properties of the gratings and an optical model (Section 5).

Alignment of each optic occurs in one of three possible "alignment positions" on the GWM (Figure la). These positions are different from the active position, but are at a known offset in $M C S R_{z}$ from the alignment position (i.e., a known number of offset steps). The alignment position for one grating corresponds to the active position for another, usually 2 or 3 positions counter-clockwise behind it. The alignment position for a given grating was selected based on the ease of viewing the zero order return from an autocollimating theodolite. The alignment position also makes the grating LCS $x$ and $y$ axes more parallel to the MCS $x$ and $y$ axes. The grating-to-be-aligned is rotated into one of these positions on the wheel before we proceed with metrology.

Each shim is sandwiched between its grating and carrier (Figure la). The shim is a triangular piece with three raised bosses that fit under flexures on the grating substrate. After making alignment measurements (Section 4.3.1), we machine the shim to adjust grating MCS $R_{x}$ and $R_{y}$ (Section 4.3.2). Since each grating is at a compound angle in the MCS (even in its alignment position), more than one iteration is usually required to bring the optic into tolerance. Measurement errors also contribute to the systematic error.

Once all gratings and the mirror are aligned and verified, the orientation of cube $\mathrm{C} 1$ in rotation and translation is measured with respect to cube $\mathrm{C} 2$ on the GWM motor mount. We make this measurement with the GWM at the home position and with the gravity vector pointing in both the MCS +y and -y directions. This "transfer" of the MCS is necessary because, when the GWM is installed to the IRMOS optical bench, $\mathrm{Cl}$ is inaccessible to our metrology equipment --- we have line of sight to $\mathrm{C} 2$ only. $^{8}$ Also, IRMOS operating condition has gravity vector pointing in the +y direction (i.e., opposite to the setup described in this paper). Any associated gravity sag is thus removed. 


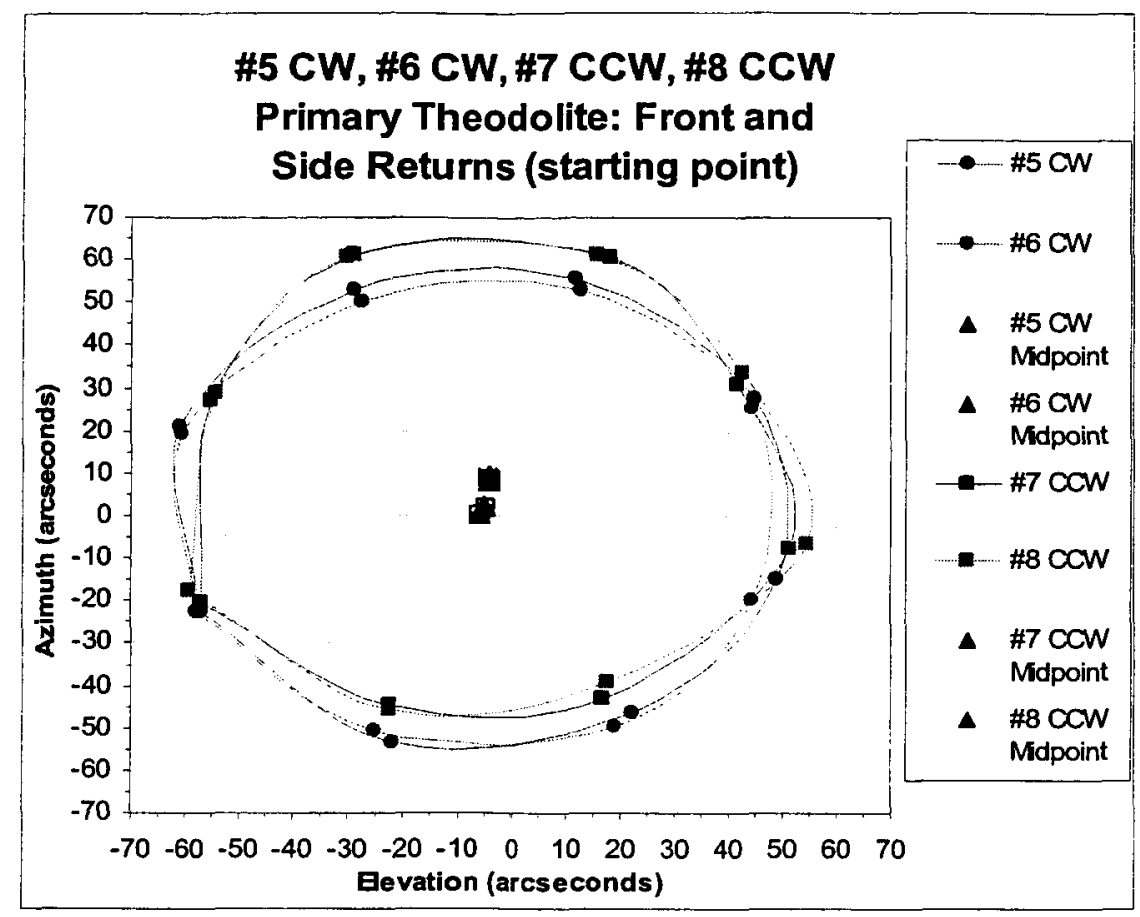

Figure 2. Sample "wobble" data ("primary" theodolite) for the front face of cube C1 for both clockwise (CW) and counterclockwise (CCW) rotations. Data are normalized to the average for each rotation. Units are arcsec. "\#n" notation refers to rotation number during the test.

\section{PRE-POPULATION CHARACTERIZATION}

\subsection{Repeatability testing}

We measure the repeatability of the unpopulated GWM in clockwise and counterclockwise MCS $R_{z}$ directions. The repeatability tolerance is $< \pm 15$ arcsec. We also make measurements of backlash (i.e., the uncertainty in MCS $R_{z}$ upon reversing direction) and wobble (i.e., the lack of repeatability of the wheel in MCS $R_{x}$ and $R_{y}$ as a function of commanded rotations in $R_{\mathbf{z}}$ ).

The test setup consists of two theodolites, both leveled with respect to gravity (elevation reference), referenced to a common azimuth origin, and focused to infinity to achieve an autocollimated return. Both theodolites are pointed at alignment cube, $\mathrm{Cl}$. $\mathrm{Cl}$ is attached to the center of the GWM. The "primary" theodolite is normal to the MCS z-face of the cube (i.e., the surface normal parallel to MCS z-axis). The "secondary" theodolite is pointed at the x-side of the cube. The test proceeds as follows: The wheel is driven one full rotation (MCS Rz) at $45^{\circ}$ increments (both clockwise and counterclockwise) for two different starting points. We record the azimuth and elevation of the autocollimating returns after each $45^{\circ}$ and $90^{\circ}$ rotation for the primary and secondary theodolites, respectively. The first starting point aligns the voltage sensor and target. The second starting point rotated the wheel such that the secondary theodolite could capture its autocollimated return from the side of the alignment cube.

\footnotetext{
- An alignment cube is an $\mathrm{Al}$ cube, $\sim 18 \mathrm{~mm}$ on a side and polished flat on five of its highly orthogonal sides. The sixth face is the interface surface. A thin, crosshair alignment fiducial is inscribed on the center of each polished face of $\mathrm{Cl}$. We assume that the sides are perfectly orthogonal and the crosshairs have zero thickness and are perfectly centered on each face of the cube.
} 
Sample repeatability data are listed in Table 2. The data are the difference in secondary theodolite elevation data for two subsequent wheel rotations at every $90^{\circ}$ (CW direction). Values greater than the \pm 15 arcsec tolerance are shown in bold.

Table 2. Sample rotational repeatability data

\begin{tabular}{|c|c|}
\hline Position (degrees) & Elevation (arcsec) \\
\hline 0 & 1.1 \\
\hline 90 & -1.7 \\
\hline 180 & -4.9 \\
\hline 270 & -16.7 \\
\hline
\end{tabular}

When CW and CCW rotations are compared, repeatability is worse. However, if the GWM is rotated in one direction only, it is generally repeatable to better than the \pm 15 arcsec tolerance.

Wobble data are shown in Figure 2. The data describe a circle, because the $\mathrm{z}$-face of $\mathrm{Cl}$ is not perfectly perpendicular to the axis of wheel rotation. The wobble is the difference in data points for a given position for a given rotation direction (CW or CCW). From Figure 2, the wobble is $\sim 5$ arcsec P-V, which is of-order the error in a theodolite measurement. However, the difference between $\mathrm{CW}$ and $\mathrm{CCW}$ direction is more significant $(\sim 10$ arcsec), but not large enough to impact IRMOS performance.

The GWM meets its repeatability specification with negligible and wobble effects. The split-gear pinion design addresses GWM backlash. Operationally, we only drive the GWM CW (Section 4.2).

\subsection{Metrology setup}

For all following work, the GWM is in the metrology setup shown in Figure 3. Hagopian et al. (1996) discuss this setup in detail. ${ }^{9}$ The scheme allows optomechanical alignment of a custom, fiducialized datum surface in six degrees of freedom using a cathetometer, a precision rotary table, and theodolites. A cube that is rigidly attached to the cathetometer and has known orientation with respect to the pointing direction of the instrument's rails serves as the laboratory azimuth reference.

The GWM is aligned to the metrology setup as follows: The steel table surface is leveled to gravity. A precision rotary table is placed on the table, on a tip/tilt plate. The rotary table's axis of rotation is aligned parallel to the gravity vector with an electronic level and checked with theodolites and a flat mirror with a highly orthogonal mounting surface. A second tip/tilt plate is attached to the top of the rotary table and roughly leveled with a bubble level. The GWM is mounted to the top of this plate and held up-right via a L-bracket fixture. The tip/tilt plate is employed to rotate the $z$ face normal of $\mathrm{Cl}$ such that it is perpendicular to gravity. The rotary table is then adjusted such that this vector is normal to the plane of the cathetometer rails (i.e., parallel to the pointing direction of the cathetometer telescope). To orient the GWM in $R_{z}$, an average normal to the side of the GWM mount is determined using a theodolite and truesquare placed on the surface. The GWM is then rotated in $R_{z}$ using the top-most tip/tilt plate until this is perpendicular to the gravity vector. Using the cathetometer, the center of the rotary table is measured and marked and the GWM is translated such that the center of the $z$-face of $\mathrm{Cl}$ (crosshair fiducial) is co-located and the MCS will not process when the rotary table spins. After the as-built MCS is defined (Section 4.2), the GWM is again aligned in rotations to the cathetometer.

\section{OPTOMECHANICAL ALIGNMENT}

\subsection{Population of carriers and gratings}

The GWM is initially aligned to the metrology setup unpopulated (i.e., carriers and gratings are not yet attached). But before carrier integration can begin, the home position must be defined. The GWM is at home when the imaging mirror is at the active position. However, placement of the target and sensor with respect to the bolt holes for carrier attachment is somewhat arbitrary. The MCS origin in $x, y, R_{x}$, and $R_{y}$ is defined as the axis of rotation, which is very close to the center of alignment cube $\mathrm{C} 1$. We orient the rotary table such that the GWM faces the cathetometer as shown in Figure 3. Assuming that the center of $\mathrm{Cl}$ is the origin and using $\mathrm{x}$ and $\mathrm{y}$ coordinates from the $\mathrm{CAD}$ model, we 
find the ideal location of bolt holes on the wheel for the active carrier with the cathetometer. The GWM is stepped to achieve the best alignment of the bolt holes and the cathetometer telescope crosshairs. Thus we find the step closest to home with respect to the sensor voltage extremum.

We attach the carriers to the wheel. We translate and rotate then in the MCS x-y plane by hand until the clear holes on the grating mounting surface line up with $\mathrm{CAD}$ model $\mathrm{x}$ and $\mathrm{y}$ targets sighted with the cathetometer. The carriers' bolts are then torqued.

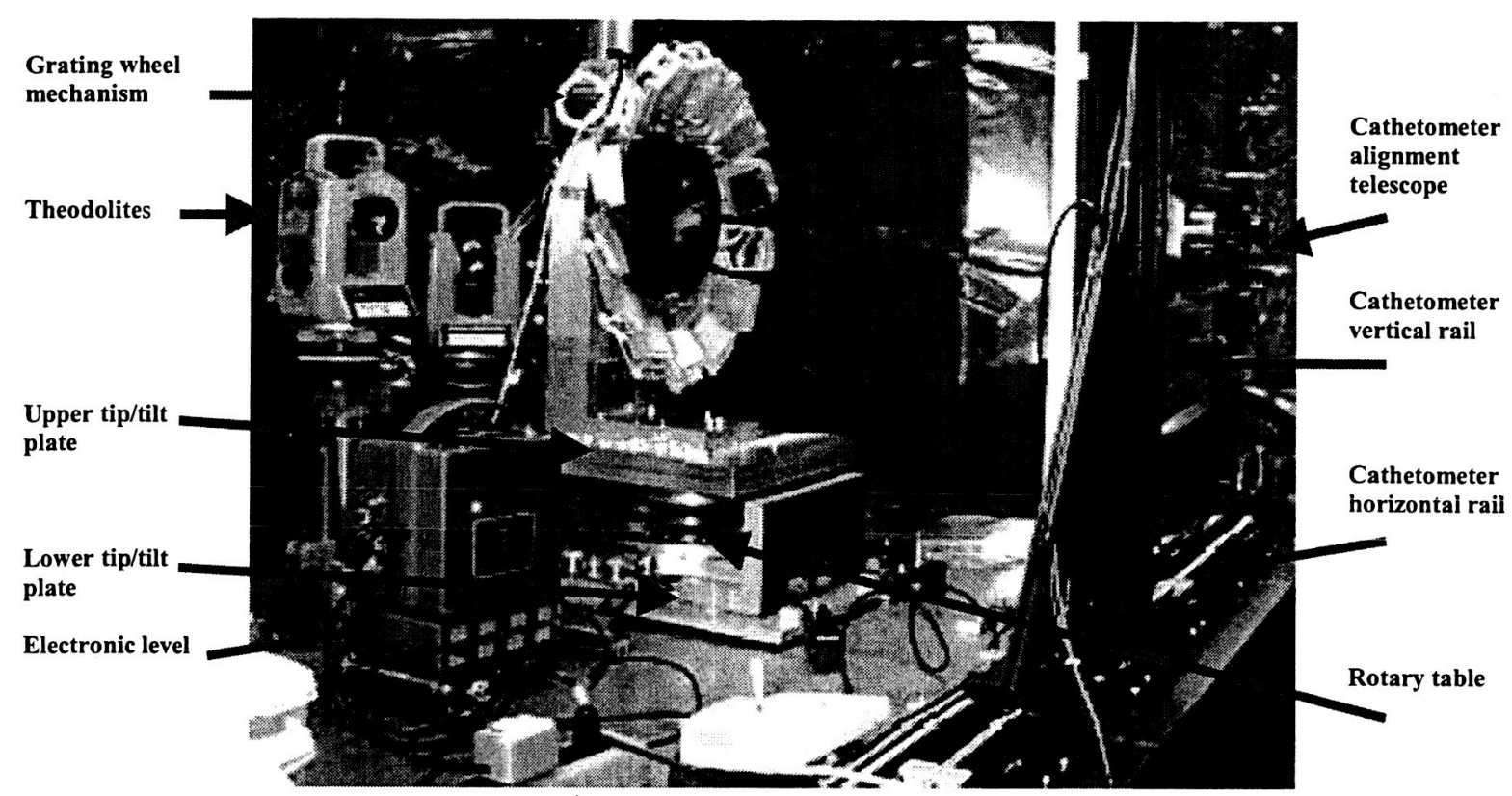

Figure 3. GWM population and alignment setup. Optics are shown covered by protective covers.

\subsection{Coordinate system definition}

The gratings and shims are attached to load the wheel before the as-built MCS is determined. The pointing direction of the MCS z-axis (thus, $\mathrm{R}_{\mathrm{x}}, \mathrm{R}_{\mathrm{y}}$ ) is referenced to the $\mathrm{z}$-face of $\mathrm{Cl}$ in a manner similar to that described for repeatability testing (Section 3.1). For each grating in its active position and for three consecutive $\mathrm{CW}$ rotations, a theodolite is used to obtain an autocollimated return from the center alignment cube $\mathrm{Cl}$, and the azimuth and elevation reading is recorded.

A plot of these data is shown in Figure 4. The data describe a circle, because the $\mathrm{z}$-face of $\mathrm{Cl}$ is not perfectly orthogonal to the axis of wheel rotation." The center of this circle is the pointing direction of the axis of wheel rotation -- the origin of the MCS in $R_{x}$ and $R_{y}$. We relate the azimuth and elevation of this point to the metrology setup (i.e., to the cube on the cathetometer).

The preferred direction of GWM rotation is CW. Changing direction slightly $(\sim 10$ arcsec) shifts the value for the pointing direction of the axis of rotation (Figure 2).

\footnotetext{
* In order to decrease the diameter of this circle, we added a $0.025 \mathrm{~mm}$-thick shim under one end of the cube mount plate.
} 
To set the MCS origin in $\mathrm{x}$ and $\mathrm{y}$, the GWM is actuated such that each grating is in the active position for three

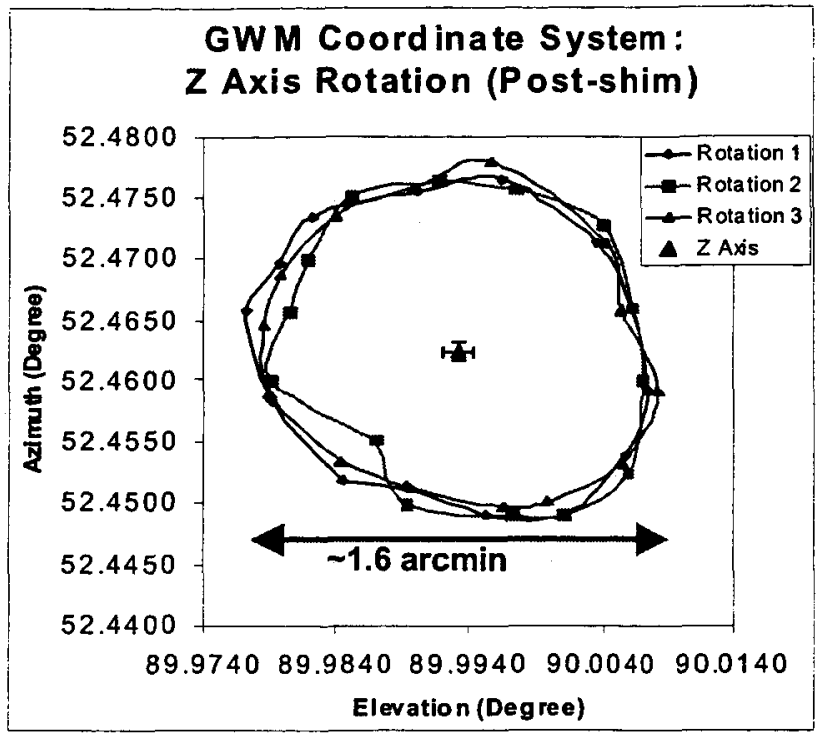

(a)

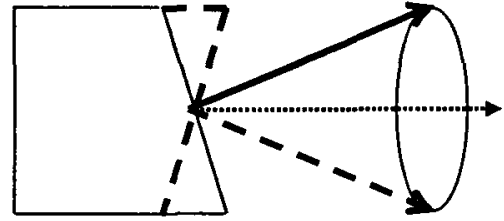

(b)

Figure 4. (a) Pointing direction of the z-axis from raw azimuth and elevation data. Units are degrees with arbitrary origin. (b) Cartoon showing the "cone" swept out by the normal to the front face of $\mathrm{Cl}$ as the GWM rotates.

complete wheel revolutions. At each active position, the cathetometer telescope crosshairs are centered on the front face fiducial of $\mathrm{Cl}$. An average value is taken for $\mathrm{x}$ and $\mathrm{y}$, and this value is defined as $\mathrm{x}=\mathrm{y}=0$. The $\mathrm{z}$ origin is defined as the center of the side fiducial of $\mathrm{Cl}$, which is visible through a gap between two carriers when the rotary table is used to present the side of the GWM to the cathetometer.

This new coordinate system data in hand, we finish alignment of the GWM to the cathetometer in $R_{x}$ and $R_{y}$, and to the center of the rotary table in $x$ ( $y$ is the vertical direction and not subject to precession). Lastly, we re-check the location of the origin in $x, y, R_{x}$, and $R_{y}$ and iterate alignment to the setup if necessary. The exact pointing direction of the MCS $x$ and $y$ axes (i.e., $R_{z}$ ) is set by location of the home position by sighting on tapped holes in the wheel for attaching the carriers (Section 4.1).

\subsection{Fine alignment of gratings}

The cathetometer is used to measure grating position in $\mathrm{x}$ and $\mathrm{y}$, and to adjust the clocking of each grating (LCS $\mathrm{R}_{\mathrm{z}}$ ) by sighting on the crosshair fiducials and comparing data to target values from the CAD model (Section 4.3.1).

For tip $\left(R_{x}\right)$, tilt $\left(R_{y}\right)$, and focus (z), two theodolites are positioned in front of the wheel. The "primary" theodolite is on the autocollimating return from the front face of $\mathrm{Cl}$, while the "secondary" theodolite is on the zero-order return from a grating in its alignment position. By relating the positions of the theodolites to each other (boresighting), a common azimuth reference is obtained and the shim is machined to align its grating to the MCS in $R_{x}, R_{y}$, and $z$ (Section 4.3.1).

\subsubsection{Alignment target calculation}

We use a $4 \times 4$, coordinate transform matrix to define the relationship between two coordinate systems. The format of a matrix is shown in Figure 5 . When a coordinate transform matrix is applied to a vector representing a point in threedimensional space, the resulting vector describes that point in the new coordinate system. The upper-left $3 \times 3$ submatrix handles rotations, while the fourth column handles translations. 
The nominal $4 \times 4$ coordinate transform matrices that would transform a vector from the MCS to each grating's LCS in the active position are obtained using the CAD instrument model (T1). We modify the nominal matrix to reflect asbuilt conditions and rotate each grating to its alignment position on the wheel. First, as-built component-level data are expressed as a matrix relative to nominal (T2). We multiply $\mathrm{T} 1$ and $\mathrm{T} 2$ to obtain the as-built matrix for the grating in the active position. Next, we transform the active position to the alignment position by using another matrix (T3; a rotation in MCS $R_{z}$ ). The final result is the transform from the MCS to the as-built LCS of the grating in the alignment position:

$$
T 4=T 1 * T 2 * T 3
$$

The final matrix, T4, is converted to laboratory measurables (e.g., target secondary theodolite azimuth and elevation) by taking the dot product of the vector of the GWM wheel rotation axis and the vector of the grating in the alignment position.
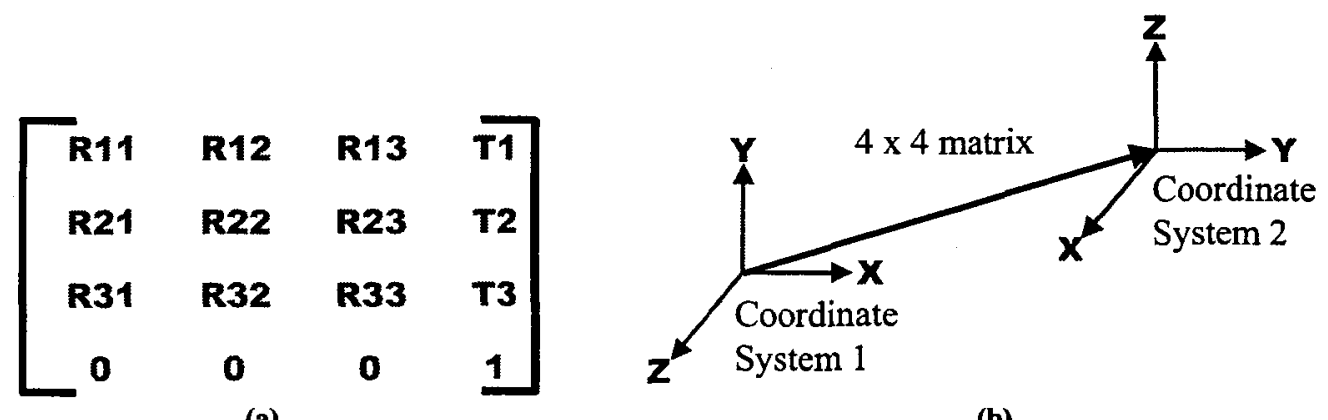

(a)

(b)

Figure 5. (a) The format of a $4 \times 4$ coordinate transformation matrix. (b) A cartoon showing two different coordinate systems.

\subsubsection{Optomechanical measurment}

The measurement technique used for fine-aligning the gratings to the GWM coordinate system is shown schematically in Figure 6. The primary theodolite is used to measure the autocollimated return from the front face of cube $\mathrm{C} 1$ (angle is shown exagerrated). Accounting of the offset between the cube normal and the z-axis (Figure 4a) is included in the data from the primary. The secondary theodolite is used to measure the zero-order return of the grating in the alignment position. The theodolites are boresighted to transfer relative azimuth information, and the rotational position of the grating in azimuth and elevation is calculated. These values are compared to those expected from the target calculation (Section 4.3.1) and a shim cut is performed. There are two pins in the carrier to ensure repeatable placement of the grating and shim on the carrier in LCS $R_{2}$ (clocking). 


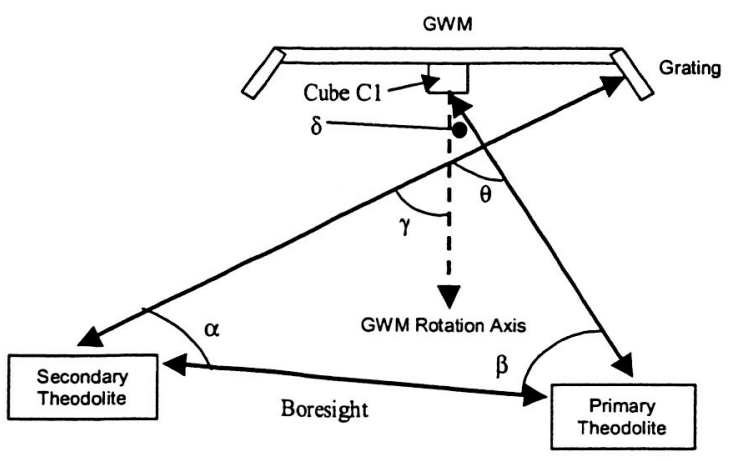

Figure 6. Schematic (top view) of the optomechanical measurement setup used for grating fine-alignment.

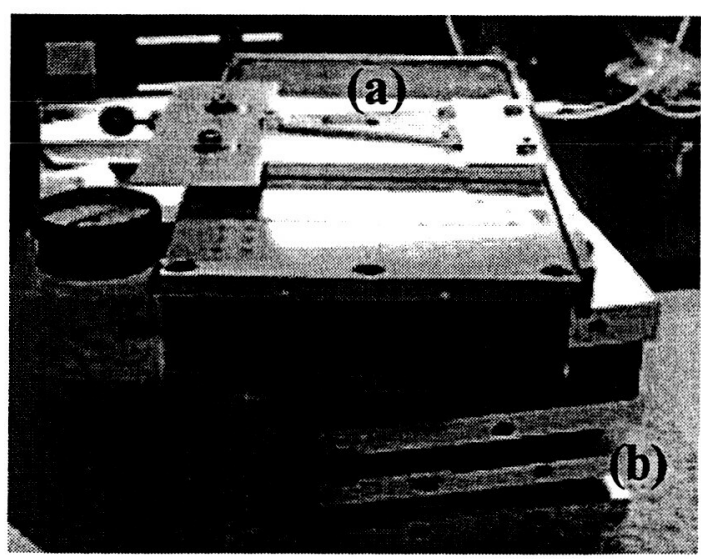

Figure 7. Shim (a) situated on sine plate (b).

\subsubsection{Shim cutting}

Shim cuts are performed with a milling machine on a $12.7 \mathrm{~cm}$ sine plate (Figure 7). The correction angle is calculated by converting the necessary adjustment values for grating angle to changes in shim thickness. The shim is clocked to approximately its MCS $R_{z}$ and pieces of shim stock are placed under the sides of the sine plate to set the angle of the plate in "azimuth" and "elevation." Finally, the grating shim is cut to the appropriate compound angle. Two iterations are usually necessary for each shim --- after each cut, the grating is remounted to the GWM and new, smaller required changes in angle are measured using the optomechanical technique discussed above. The first cut aligns the grating in azimuth and elevation and the second cut aligns the grating in despace and any residual necessary azimuth and elevation correction. After alignment, the grating bolts are torqued.

\subsubsection{Alignment results}

Final alignment offsets from nominal for each optic are given in Table 3. Bold values represent out of tolerance values for which a performance wavier is granted.

\section{ALIGNMENT VERIFICATION}

We use the properties of the front (diffractive/reflective) surface of the gratings/mirror to verify alignment. The alignment verification setup consists of two theodolites, a cathetometer, a helium-neon laser $(\lambda=632.8$ $\mathrm{nm}$ ) with neutral-density filters, a ground glass sheet, and the GWM with all gratings populated and aligned. We shine the laser at the surface of a grating and measure the location of the diffracted orders in the metrology setup. ${ }^{10}$

The ground glass sheet is aligned to the plane of the cathetometer rails using the cathetometer telescope in autocollimation. The ground glass sheet is adjusted in azimuth and elevation such that the autocollimated return is centered on the crosshairs in the telescope. The laser is positioned such that it is incident and 
roughly centered on the grating to be verified. Because the glass sheet is already aligned to the cathetometer, the rotary table is rotated into a position where diffracted spots are visible to the cathetometer on the glass sheet. The procedure is:

1. Theodolites are positioned such that one measures the angle of the laser beam and the other measures the autocollimating return from the front $(z)$ face of $\mathrm{Cl}$. Spin rotary table such that the GWM is out of the line of sight of the primary theodolite to the laser, but such that the secondary theodolite may obtain a return from $\mathrm{Cl}$.

2. Record the laser azimuth and elevation. Capture the autocollimating return from the front face of $\mathrm{Cl}$ with the secondary theodolite and record azimuth and elevation. Boresight the theodolites.

3. Actuate the GWM such that the grating of interest is in the alignment verification position.

4. Face GWM such that it is face-on to the plane of the cathetometer.

5. Spin the rotary table until the laser is incident on the grating, producing a diffractive pattern on the ground glass sheet. Record rotary table position.

6. Position the cathetometer telescope such that the crosshairs are aligned with the centroid of the spot produced by the incident beam on the grating. Record $\mathrm{x}$ and $\mathrm{y}$ positions.

7. Repeat step six until the positions of several diffracted spots are recorded. The ground glass shows orders from one to 26 , depending on the grating.

8. Repeat for all gratings.

9. Fit a line to the $x$ and $y$ positions of the diffracted orders for each grating. The angle of this line is the clocking angle of the grating in the metrology setup. Compare it to the angle predicted by a computer model of the setup.

These data are consistent with model predictions to within alignment tolerances and the systematic and statistical error inherent in the setup and measurement.

Table 3. Alignment conditions based on fiducial measurement (MCS)

\begin{tabular}{|c|c|c|c|c|c|c|}
\hline Grating \# & $\begin{array}{l}\text { Azimuth } \\
\text { (arcsec) }\end{array}$ & $\begin{array}{c}\text { Elevation } \\
\text { (arcsec) }\end{array}$ & $\begin{array}{l}\text { Clocking } \\
\text { (arcmin) }\end{array}$ & $X(\mathrm{~mm})$ & $Y(\mathrm{~mm})$ & $\mathrm{Z}(\mathrm{mm})$ \\
\hline Tolerances & \pm 1 arcmin & \pm 1 arcmin & \pm 6 arcmin & $\pm 1 \mathrm{~mm}$ & $\pm 1 \mathrm{~mm}$ & $\pm 2 \mathrm{~mm}$ \\
\hline 1 & 53 & -11 & 7.005 & 0.51 & 0.20 & -1.39 \\
\hline 2 & -83.6 & -2.7 & -3.00 & 0.94 & 0.09 & -1.40 \\
\hline 3 & 13.9 & 3.1 & -2.014 & 0.13 & 0.41 & -1.24 \\
\hline 4 & 20.8 & 4.7 & 1.006 & -0.18 & 0.22 & -1.50 \\
\hline 5 & 49.0 & -39.6 & -0.01 & 0.49 & 0.47 & -1.44 \\
\hline 6 & -39.4 & 47.5 & 7.01 & 0.89 & -0.10 & -1.61 \\
\hline 7 & 7.3 & 16.0 & 4.008 & -0.29 & -0.14 & -1.40 \\
\hline 8 & -18.5 & -1.0 & 0.002 & 0.53 & -0.001 & -1.63 \\
\hline 9 & 45.3 & -8.8 & -2.208 & -0.44 & 0.15 & -1.25 \\
\hline 10 & 6.7 & 50.7 & 2.002 & 0.25 & 0.16 & -1.20 \\
\hline 11 & 26.6 & 52.2 & 8.01 & -0.24 & -0.04 & -1.27 \\
\hline 12 & 34.7 & -47.4 & 0.015 & 0.26 & 0.31 & -1.59 \\
\hline 13 & 25.7 & -5.4 & 3.005 & 0.37 & 0.22 & -1.39 \\
\hline 14 & -51.4 & -8.1 & -3.70 & 0.02 & 0.16 & -0.50 \\
\hline
\end{tabular}

\footnotetext{
- Approximate measurement errors are \pm 5 arcsec for azimuth and elevation, \pm 30 arcsec for clocking, and $\pm 0.025 \mathrm{~mm}$ for $\mathrm{x}, \mathrm{y}$, and $\mathrm{z}$.
} 


\section{CONCLUSION}

The IRMOS GWM is fully integrated and ready for cryogenic operation. The GWM is integrated with the IRMOS bench and images obtained at ambient are consistent with an instrument that is aligned within tolerance.

Note: Weeks after integration with the instrument bench, a mechanical repair to the GWM required us to rebuild the metrology setup, realign the GWM to it, and check the locations of the fiducials with respect to the MCS. We found two unanticipated differences in grating and mirror location. To bring the optics into nominal alignment, we had to rotate the wheel one step $C W\left(-R_{z}\right)$, then translate it in $+x$ by $\sim 1 \mathrm{~mm}$. Possible reasons for the $R_{z}$ offset include a dropped step (electronics/software issue) and a systematic alignment error --- the MCS origin in $R_{z}$ is somewhat arbitrarily defined (Section 4.2). This degree of freedom was carefully re-referenced prior to re-integration with the bench. The $+x$ offset is most likely caused by a failure to double-check the measurement of the center of the rotary table (Section 3.2).

\section{ACKNOWLEDGEMENTS}

For technical support and useful discussions, we gratefully acknowledge: Claef F. Hakun, Linette D. Kolos, Carl R. Strojny, and Geraldine A. Wright of GSFC; Thomas J. French, Joesph C. McMann, and Louis R. Worrel of Management Technology International Corp. at GSFC; and Eric Hochberg of the Jet Propulsion Laboratory. This work is supported by the JWST project at GSFC and the Center Director's Discretionary Fund at GSFC and Director's Discretionary Fund at STScI.

\section{REFERENCES}

1. J. W. MacKenty, M. A. Greenhouse, R. F. Green, L. M. Sparr, R. G. Ohl, R. S. Winsor and the IRMOS team, "IRMOS: An Infrared Multi-Object Spectrometer using a MEMS micro-mirror array," Proc. SPIE 4841, pp. 953-961, 2003.

2. R. Winsor, J. W. MacKenty, M. Stiavelli, M. Greenhouse, E. Mentzell, and R. Ohl, "Optical design for an Infrared Multi-Object Spectrometer (IRMOS)," Proc, SPIE 4092, pp. 102-108, 2000.

3. J. P. Schepis, M. McClendon, M. Webb, J. Hylan, and J. W. MacKenty, "Two Cryogenic Mechanisms for Earth Based Infrared Astronomy," Proceedings of the $10^{\text {th }}$ European Space Mechanisms and Tribology Symposium, 2003 (accepted).

4. R. W. Toland, R. G. Ohl, M. P. Bathelmy, S. W. Zewari, M. A. Greenhouse, and J. W. MacKenty, "Effects of forged stock and pure aluminum coating on cryogenic performance of heat treated aluminum mirrors," Proc. SPIE 5172, 2003 (this volume).

5. R. G. Ohl, W. Preuss, A. Sohn, S. Conkey, K. P. Garrard, J. G. Hagopian, J. M. Howard, J. E. Hylan, S. M. Irish, J. E. Mentzell, M. Schroeder, L. M. Sparr, R. S. Winsor, S. W. Zewari, M. A. Greenhouse, and J. W. MacKenty, "Design and fabrication of diamond machined, aspheric mirrors for ground-based, near-IR astronomy," Proc. SPIE 4841, pp. 677-688, 2003.

6. S. Conkey, "Surface distortion of high precision optics due to assembly loads using FEA," Innovative FEM Solutions to Challenging Problems, Finite Element Modeling Continuous Improvement Workshop 2001, J. Loughlin, coordinator, NASA/Goddard Space Flight Center, May 16-17, 2001.

7. J. J. Lyons III and J. J. Zaniewski, "Process for polishing bare aluminum to high optical quality," NASA Tech Briefs 23, pp. 58-59, 2001.

8. J. A. Connelly, R. G. Ohl, J. E. Mentzell, T. J. Madison, J. E. Hylan, R. G. Mink, T. T. Saha, J. L. Tveekrem, L. M. Sparr, V. J. Chambers, M. A. Greenhouse, and J. W. MacKenty, "Alignment and performance of the Infrared Multi-Object Spectrometer," Proc. SPIE 5172, 2003 (this volume).

9. J. G. Hagopian, P. A. Hayes, J. A. Crooke, and J. J. Lyons, "Opto-mechanical alignment of the Composite Infrared Spectrometer (CIRS) for the Cassini mission to Saturn," Proc, SPIE 2814, pp. 46-58, 1996.

10. E. Hochberg, "System for Measuring Three Tilts and Distance of an Object." NASA Tech Briefs, November 2001. 